\title{
Cancer Surgery and COVID19
}

\section{The COVID19 Subcommittee of the O.R. Executive Committee at Memorial Sloan Kettering}

Departments of Surgery, Anesthesia and Critical Care Medicine, Neurosurgery and Nursing, Memorial Sloan Kettering Cancer Center, New York, NY

The COVID19 pandemic has stressed health care systems around the world and presents particular challenges to cancer surgeons and cancer surgery programs. This article describes our real-time experience dealing with some of these challenges.

New York City was not the first city in the United States to see a blossoming of COVID19 cases, but it has had the most rapid increase in case numbers and currently has the most cases overall. Our city has been described accurately as the epicenter of the pandemic in this country. Currently, some other institutions are dealing with the same challenges we are facing, and many others undoubtedly will face these challenges in the weeks and months to come.

Although Memorial Sloan Kettering (MSK) is a 500-plus-bed dedicated cancer hospital rather than a general hospital, we will try to emphasize challenges that will face all cancer surgery programs such as the following: What is elective surgery, and what is elective cancer surgery? What are the operating room (OR) safety challenges facing cancer surgeons and operating room teams, and what can be done to minimize these? What are our obligations to our patients versus our societal obligations to preserve hospital beds, personal protective equipment (PPE), and ICU/ventilator resources for potential COVID19 patients? And what can surgical leaders do to prepare their faculty and staff and to assist their institutions in facing these challenges? The information presented in this article represents the collective views of the authors and does not represent an institutional position.

The collaborators for the COVID19 Subcommittee of the Operating Room Executive Committee are listed in the Acknowledgments.

(C) Society of Surgical Oncology 2020

First Received: 1 April 2020;

Published Online: 13 April 2020

\section{IS SCHEDULED CANCER SURGERY ELECTIVE SURGERY?}

We initially faced this question when notified that the state and local government wanted all hospitals to cancel elective surgery in preparation for a surge of COVID19 patients. A similar recommendation from the American College of Surgeons (ACS) appeared shortly afterward.

At MSK, our surgeons manage approximately 30,000 cases annually in one of 13 Department of Surgery services (Breast, Colorectal, Dental, Gastric and Mixed Tumor, Gynecology, Head and Neck, Hepato-Pancreatico-Biliary, Ophthalmologic Oncology, Orthopedic, Pediatric Surgery, Plastic Surgery, Thoracic, and Urology) and the Department of Neurosurgery.

Our immediate response to the edict calling for the cancellation of elective surgery was to distinguish elective surgery from potentially curative cancer surgery, which we have called "essential cancer surgery." Approximately $5-10 \%$ of our surgical volume consists of cases that are truly elective (e.g., incisional hernia repairs, cholecystectomy for biliary colic, ostomy takedowns, and some plastic/reconstructive procedures), and another $20-30 \%$ of our cases are more cancer-specific but can safely be deferred for several months (including prostatectomy for low-grade prostate cancer, pancreatectomy for cystic neoplasms without cancer or high-risk features, and thyroidectomy for low-grade thyroid malignancies). We initially moved these cases off our schedule in compliance with the governmental edicts while continuing to perform essential cancer surgical procedures such as brain tumor, breast, colon, stomach, pancreas, liver, bladder, kidney, and lung resections. Subsequent guidance from the ACS has validated this approach (https://www.facs.org/about-ac s/covid-19/information-for-surgeons/triage), and the NY 
State Department of Health has endorsed this position. Our rules for choosing patients to undergo surgery are outlined in Table 1.

In framing our institutional response to a potentially escalating number of COVID19 patients, we planned a stepwise reduction in surgical activity based on competing needs for beds, ORs, and ICUs/ventilators. We also recognized that staff illness and quarantine requirements together with critical supply shortages had the potential to limit our ability to perform essential cancer surgery even with sufficient ORs and beds. Our planning ranged from continuing to manage the full range of essential cases while taking some ORs off line due to the cancellation of elective and nonessential surgery, to reducing activity by $25 \%$, $50 \%$, or $75 \%$ due to limitations of resources being diverted to COVID19 care, to closing all scheduled ORs and recovery space and devoting most of our anesthesia machines to use as auxiliary ventilators while preserving a small number of ORs for true surgical emergencies.

The unpredictable nature of the prospective "surge" in COVID19 patients, at least currently, has caused us to try to tread the line between unnecessarily cancelling essential surgical procedures while the hospital has ample bed and staff capacity and not being ready to pivot to postponing cases as a COVID19 surge materializes.

The Operating Room Executive Committee, consisting of leaders from Surgery, Anesthesia, and Nursing, meets twice daily to review the hospital census, bed and ICU availability, COVID19 activity at our institution and in the city, and requests for case scheduling. All cases are scrutinized to determine whether they are truly essential. When necessary, individual surgeons are contacted to justify medical necessity. We also have shortened the time for definitive scheduling of cases. Our current expeditious scheduling in $48-72 \mathrm{~h}$ will allow surgical volume to be ramped down quickly if necessary. Service chiefs have been asked to review the weekly schedules for their services and to prioritize a triage list for use in the event that we need to cancel even some essential cases. We believe this approach recognizes our primary mission to treat cancer patients while allowing us to be ready to deal with an increasing volume of COVID19 patients as they arrive.

\section{WHAT ARE THE OPERATING ROOM SAFETY CHALLENGES THAT FACE SURGEONS, ANESTHESIOLOGISTS, AND PERIOPERATIVE STAFF?}

Recommendations from the Centers for Disease Control and Prevention (CDC) and professional organizations for best practices to reduce risk to care providers have continued to evolve as the pandemic has unfolded. An ideal solution would be to test all patients immediately before surgery and postpone procedures for COVID19-infected patients. However, limitations on testing capacity may make this approach impossible. Because droplet-based transmission is a primary means of COVID19 spread, procedures involving the airway, including intubation, may place anesthesiology staff and surgeons at particularly high risk.

To maximize protection of staff in the OR, anesthesiology staff are provided N95 masks for all procedures. Due to the shortage of N95 masks, other OR staff are asked either to vacate the OR or to maintain a distance of at least 6 feet during induction and extubation.

Head and neck, thoracic, and neurosurgeons may face particularly high risks for certain procedures, and chiefs in each of these disciplines have worked to minimize servicespecific high-risk procedures. Guidelines have been created for high-risk procedures that must be followed for these services, including the selective use of preoperative testing (with postponement of COVID19-infected patients), the standard use of N95 masks and face shields or goggles, and selective use of filtered positive-pressure airflow helmets.

TABLE 1 Memorial Sloan Kettering Cancer Center (MSK) guidelines for surgery

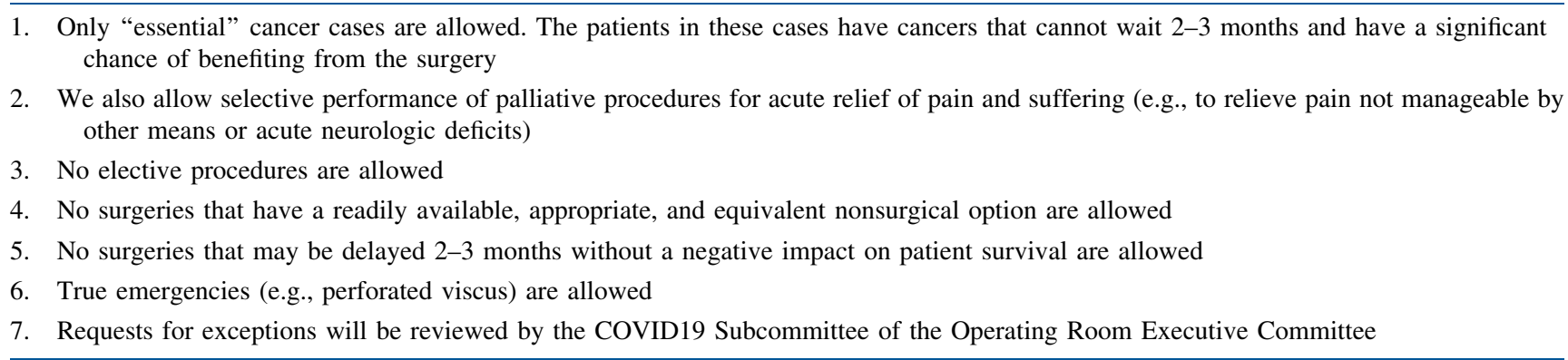




\section{PATIENT-CARE MISSION VERSUS SOCIETAL OBLIGATIONS}

All physicians have a responsibility to provide the best care to their patients. The COVID19 pandemic creates competing and, in some cases, opposing institutional and societal responsibilities with regard to health care provider safety, use of PPE, and allocation of hospital beds and ventilators. Surgical oncologists may be placed in a position of deciding whether health benefits, and even the chance of survival, for their individual patients potentially able to undergo essential cancer surgery, justify the risk to themselves and other health care providers and the use of limited resources.

In terms of health care provider risk, this is not the first nor the last epidemic or pandemic to place individuals at risk. The fact that a similar event has not occurred in the United States for many years makes this pandemic more frightening, although for those of us who practiced or trained in the early years of the human immunodeficiency virus (HIV) epidemic, the sudden need for concern about personal safety is not dissimilar. The examples of health care worker morbidity and mortality from around the world should certainly give us pause, recognizing that environments in which these have been most common are those unable to provide adequate PPE and that social transmission in high-prevalence geographic areas likely surpasses nosocomial transmission as a risk factor.

It also is important to recognize that even if COVID19 mortality in the United States reaches the level of the most severe projections, more cancer deaths than COVID19-related deaths will occur in the next year and that in the absence of appropriate care, the ratio of cancer mortality to COVID19 mortality would be even greater. Until a vaccine is developed or sufficient population infection develops to achieve "herd immunity," COVID19 is likely to persist for at least a number of months. In the meantime, patients whose essential cancer surgery is postponed until the pandemic is behind us face a greater risk of disease-related mortality. Therefore, abandoning cancer care and focusing only on COVID19 care appears to be an illogical societal choice that will have a negative impact on cancer patients.

In the attempt to provide essential cancer surgery for the patients who need it, all possible steps must be taken to minimize health care provider risk. Ultimately, this could include preoperative testing for all surgical patients, although the limited availability of such testing currently hampers this approach at many locations. Other steps that can be taken currently include scheduling only patients who definitely require surgical care per the guidelines listed in Table 1, providing appropriate PPE to everybody at risk, and minimizing provider exposure by carefully selecting and, whenever possible, performing preoperative
COVID19 testing for patients undergoing procedures that will place providers at the highest risk. Managing OR schedules to fill fewer rooms that run longer through the day and minimizing the number of staff on site constitute another strategy that can reduce staff exposure and risk.

The appropriate preservation of limited resources such as PPE, hospital beds, and particularly ventilators represents another series of decisions that may place individual cancer patient surgery interests in conflict with the locoregional response to COVID19. Most hospitals have traditionally held relatively modest reserves of PPE, depending on just-in-time purchasing strategies to manage inventory. The increased demands for PPE, particularly N95 masks, and the closure of some traditional supply chains is straining PPE supplies in many institutions. To date, the demand for hospital beds and ventilators is not critical in the United States, but is projected to become critical very shortly in a number of regions, including ours. The international examples of patient demand outpacing PPE and ventilator resources are a sobering reminder that preserving these scarce resources before the projected peak in COVID19 activity may be important in minimizing subsequent provider and patient mortality.

There is no simple answer to the question of trading off patient needs against provider risk and institutional and societal resource utilization. In our view, this begins with individual surgeons who need to try as much as possible to balance their ethical responsibilities to individual patients with the current need to minimize caregiver risk and resource utilization. Leaders at each institution need to oversee this process with a careful eye on appropriateness of planned surgery, trends in locoregional disease activity, and the availability and supply of institutional resources.

\section{WHAT ROLE(S) SHOULD SURGEON LEADERSHIP PLAY IN OPERATING ROOM DECISION-MAKING AND INSTITUTIONAL PREPARATIONS FOR THE COVID19 PANDEMIC?}

The COVID19 pandemic represents a novel challenge to the leadership at each institution. No one has experienced anything like this before. Our approach has been to closely involve the surgeon, anesthesia, and nursing leaders on our OR Executive Committee in managing OR scheduling on a twice-daily basis with regard to the appropriateness of individual cases, the availability of nursing and anesthesia staff to perform the projected case volume, and more broadly, the review of the impact that OR activity has on patient bed and resource utilization. The recommendations of this group are coordinated with institutional planning and decision-making for our institution by our Hospital Incident Command System, which includes a 
representative from our committee together with other medical, surgical, and administrative hospital leadership and also meets twice daily.

To date, clear paths of communication and information flow have been vital in our institutional response, which is ongoing. Electronic communication from the COVID19 Subcommittee to all departmental and perioperative staff has been carried out on a regular basis so that the entire perioperative team, including support staff, receive a cohesive and consistent message. We hope that the steps we have taken will allow us to optimize the care of our cancer patients and our COVID19 patients while minimizing risk to staff and appropriately conserving patient care resources. A review of our successes, failures, and lessons learned may be the subject of a future editorial.
ACKNOWLEDGMENT The COVID19 Subcommittee of the Operating Room Executive Committee Collaborators include Laura Ardizzone (ardizzol@mskcc.org), Thomas Barber (barbert@mskcc.org), Jeffrey Drebin (drebinj@mskcc.org), Gregory Fischer (fischerg@mskcc.org), Elizabeth Jewell (jewelle@mskcc.org), Vincent Laudone (laudonev@mskcc.org), Marcia Levine (levinem@mskcc.org), Jeannine Linder (riveraj4@mskcc.org), Brett Simon (simonb1@mskcc.org), Christopher Stromblad (stromblc@mskcc.org), Viviane Tabar (tabarv@mskcc.org), Martin Weiser (weiser1@mskcc.org), and Shok-Jean Yee (yees@mskcc.org).

DISCLOSURE There are no conflict of interest.

Publisher's Note Springer Nature remains neutral with regard to jurisdictional claims in published maps and institutional affiliations. 